\title{
Structural and Functional Analysis of a PadR-like Transcription Factor From Bacteroides fragilis
}

\author{
Choongdeok Lee, Meong il Kim, Jaewan Park and Minsun Hong \\ Division of Biological Science and Technology, Yonsei University, Wonju 26493, Republic of Korea
}

A phonolic acid decarboxylase (padC) regulator, PadR was initially identified in grampositive bacteria Pediococcus pentosaceus. PadR is a transcription factor that regulates the expression of the phenolic acid decarboxylase enzyme in bacteria. In last 18 years, numbers of PadR homologs and PadR-like proteins have been grown and found in many other bacteria to become the PadR family. In bacteria, the PadR family members play various roles that include detoxification, drug transports and circadian rhythms regulation. Despite a growing interest in the PadR family, the biophysical and biochemical data to suggest regulatory mechanism of the PadR proteins are very limited. Thus, we performed a structure-based functional analysis using a PadR-like protein from Bacteroides fragilis (BfPadR). We will present a crystal structure of BfPadR at $2.5 \AA$ resolution. Moreover, as a putative transcriptional regulator, an operator site for BfPadR will be addressed to propose a DNA binding model. 